\title{
Long run forward rates and long yields of bonds and options in heterogeneous equilibria
}

\section{Journal Article}

Author(s):

Malamud, Semyon

Publication date:

2008

Permanent link:

https://doi.org/10.3929/ethz-b-000004715

Rights / license:

In Copyright - Non-Commercial Use Permitted

Originally published in:

Finance and Stochastics 12(2), https://doi.org/10.1007/s00780-007-0058-0 


\title{
Long run forward rates and long yields of bonds and options in heterogeneous equilibria
}

\author{
Semyon Malamud
}

Received: 25 July 2007 / Accepted: 22 October 2007 / Published online: 13 December 2007

(C) Springer-Verlag 2007

\begin{abstract}
We prove that, in a heterogeneous economy with scale-invariant utilities, the yield of a long term bond is determined by the agent with maximal expected marginal utility. We also prove that the same result holds for the long term forward rates.

Furthermore, we apply Cramér's large deviations theorem to calculate the yield of a long term European call option. It turns out that there is a threshold risk aversion such that the option yield is independent of the risk aversion when the latter is above the threshold. Surprisingly, the long term option yield is always greater than or equal to the corresponding equity return. That is, in the long run, it is more profitable to buy a long maturity call option on equity than the equity itself.
\end{abstract}

Keywords Heterogeneity · Asset prices · Yield curve · Forward rates · Option

Mathematics Subject Classification (2000) 91B28 · 91B60 - 91B64 · 91B70

JEL Classification D91 $\cdot$ E43 - G12

\section{Introduction}

In this paper, we study long run zero coupon bond yields and forward rates in heterogeneous, complete market economies. In equilibrium, long run forward rates and long yields on bonds reflect the price of long run economic risks and, for this reason, help us in understanding the role of these risks. See, for example, Alvarez and Jermann [2] and Dybvig et al. [10] (see also Hubalek et al. [12] for a general mathematical result).

S. Malamud $(\varangle)$

D-MATH, ETH Zürich, Rämistrasse 101, 8092 Zürich, Switzerland

e-mail: semka@math.ethz.ch 
There is a large literature analyzing asset prices in heterogeneous economies. See, e.g., Dumas [9], Ross [18], Wang [20, 21], Constantinides and Duffie [5], and Gollier and Zeckhauser [11]. A direct predecessor of our paper is the paper of Wang [22]. Wang considered an economy populated by two agents with CRRA utility functions, identical discount factors, and risk aversions 1 and 0.5 and proved that the long run zero coupon bond yield is determined by the agent with the largest expected marginal utility. In this paper, we prove this result for an arbitrary number of agents with arbitrary heterogeneous discount factors and risk aversions. Furthermore, we prove a much stronger statement: an analogous result holds for long run forward rates. Our results can be directly extended to any scale-invariant preferences, including heterogeneous beliefs (such as Wang [20] and Wang [21]) and state-dependent preferences, generated by habit formation (such as, e.g., Constantinides [4] and Abel [1]). Even though we work in discrete time, all our results literally hold for continuous time economies. Note also that our result extends Lengwiler [14], who considered an economy with heterogeneous discount factors and proved that the long run bond yield is determined by the most patient agent.

It is well known that, in a homogeneous economy with standard, CRRA preferences and geometric random walk (Brownian motion) aggregate endowment, the equity price is proportional to the aggregate endowment and, consequently, options are priced via the standard Black-Scholes formula. Thus, the Black-Scholes formula can be considered as a trivial, homogeneous special case of our heterogeneous equilibria. Introducing heterogeneity is a natural way of generating new effects, such as stochastic volatility. For example, Benninga and Mayshar [3] considered a one-period economy with heterogeneous risk aversions and discount factors and showed how heterogeneity generates a correction to the Black-Scholes formula and, in particular, is able to produce smiles and skews compatible with real data.

In this paper, we study for the first time the yield of a long maturity call option, that is, the per-period return on holding the option up to maturity. We explicitly calculate the limit of the option yield as the maturity tends to infinity. Several surprising phenomena arise. First, there is a threshold risk aversion such that the long run option yield is independent of the risk aversion when the latter is above the threshold. Second, the option yield is always greater than or equal to the corresponding equity return. That is, in the long run, it is more profitable to invest in options than in equities. It would be very interesting to compare these theoretical predictions with real data.

It is necessary to point out that the word "agent" is used to denote a set of identical agents, each of measure zero. Of course, an agent of positive measure cannot remain a price taker when he begins to dominate certain asset returns at sufficiently long horizon. ${ }^{1}$ However, we abuse this fine distinction and use the word "agent" alone.

\section{The model}

We assume a discrete-time, pure exchange economy with a single, perishable consumption good (numeraire). The aggregate endowment stream $W_{t}, t \geq 1$, equiva-

\footnotetext{
${ }^{1}$ We thank Rajnish Mehra for this important remark.
} 
lently, the single good stream, is a geometric random walk normalized by $W_{0}=1$. That is, there exists a sequence of positive, i.i.d. random variables $X_{t}$ such that

$$
W_{t}=X_{1} \cdots X_{t}
$$

for all $t$. The information structure is encoded in the filtration $\left(\mathcal{F}_{t}, t \geq 0\right)$ on the underlying probability space $(\Omega, \mathcal{B}, P)$ generated by the aggregate endowment process $\left(W_{t}\right)$. We emphasize that all our results can be directly extended to continuous time by incorporating the model of [22].

We also make the common assumption that there is a "money market" in which a one-period risk-free bond can be traded at each moment of time. Agents trade competitively in both the equity and money markets and consume the proceeds. Furthermore, we assume that the market is dynamically complete. This assumption is naturally fulfilled in standard, Brownian motion driven complete markets (see, e.g., [13]). When time is discrete, things become slightly different, because there may be exceptional situations for a set of parameters of measure zero for which the dimension of the market subspace falls down. But for risky assets with generic endowment processes, this does not happen. The standard way to proceed is the following: first assume that the market is complete and calculate the equilibrium state price densities. Then, find the generic set of (exogeneously specified) risky dividend processes that complete the market. This set will be the complement of a countable set of hyperplanes.

Note that in discrete time with a finite time horizon, we need infinitely many assets to complete the market if the probability space $\Omega$ is infinite. But since our proofs and results do not depend on the discreteness of time, we present all the arguments for general probability spaces.

Since the market is dynamically complete, it is well known (see, e.g., $[8,13])$ that there exists a unique positive state price density process $\mathbf{M}=\left(M_{t}, t \geq 0\right)$ (normalized by $M_{0}=1$ ) through which all securities can be priced. In particular, the price of the Lucas tree equity, whose dividend process coincides with the aggregate endowment $\left(W_{t}\right)$, is given by

$$
P_{t}^{\mathbf{W}}=E_{t}\left[\sum_{\tau=1}^{\infty} \frac{M_{t+\tau}}{M_{t}} W_{t+\tau}\right]
$$

(we assume no bubbles). Abel [1] suggested considering equities with dividend processes $\left(W_{t}^{\alpha}\right)$ with $\alpha \in \mathbb{R}$, along with the Lucas tree asset. The parameter $\alpha$ is introduced to account for leverage effects. In general, the price $P_{t}^{\mathbf{D}}$ at time $t$ of an asset with a dividend process $\mathbf{D}=\left(D_{t}, t \geq 0\right)$ is given by

$$
P_{t}^{\mathbf{D}}=E_{t}\left[\sum_{\tau=1}^{\infty} \frac{M_{t+\tau}}{M_{t}} D_{t+\tau}\right] .
$$

Furthermore, the price at time $t_{1}$ of a risk-free zero coupon bond maturing at time $t_{2}$ is given by

$$
B^{\mathrm{F}}\left(t_{1}, t_{2}\right)=E_{t_{1}}\left[\frac{M_{t_{2}}}{M_{t_{1}}}\right] .
$$


That is, specifying asset prices is equivalent to specifying the state price density process. For the Lucas tree equity price to be defined, it is necessary that the state price densities lie in the natural price space

$$
l_{1}(\mathbf{W})=\left\{\mathbf{M}: \sum_{t=0}^{\infty} E\left[W_{t}\left|M_{t}\right|\right]<\infty\right\}
$$

The economy is populated by $n$ classes of identical CRRA agents. Since they aggregate (see [19]), we will use the name agent $i$ for the representative agent of a class $i, i=1, \ldots, n$. We denote by $N=\{1, \ldots, n\}$ the set of all agents.

Agent $i$ has a constant relative risk aversion (CRRA) utility function. He chooses his random consumption $x_{i t}$ at each time $t \geq 0$ and each possible state of the world to maximize the expected discounted intertemporal utility function ${ }^{2}$

$$
E\left[\sum_{t=0}^{\infty} \delta_{i}^{t} \frac{x_{i t}^{1-\gamma_{i}}-1}{1-\gamma_{i}}\right]
$$

Here, $\gamma_{i}$ is the relative risk aversion of agent $i$, and $\delta_{i}$ is his discount factor (patience). Agent $i$ is endowed with $\eta_{i}$ shares of equity, and $\sum_{i=1}^{n} \eta_{i}=1$ since we normalize the supply to be one. An agent finances his consumption by trading assets. It is easy to show (see, e.g., Wang $[8,22]$ ) that, since markets are complete, the set of feasible consumption streams (the budget set) of agent $i$ can be easily described in terms of the unique state price density process. Agent $i$ chooses his optimal consumption stream $\left(x_{i t}\right)_{t \geq 0}$ from the corresponding consumption space

$$
l_{1}(\mathbf{M})=\left\{\left(x_{i t}\right)_{t \geq 0}: \sum_{t=0}^{\infty} E\left[x_{i t} M_{t}\right]<\infty\right\}
$$

satisfying the budget constraint

$$
E\left[\sum_{t=0}^{\infty} x_{i t} M_{t}\right]=\eta_{i} E\left[\sum_{t=0}^{\infty} W_{t} M_{t}\right]
$$

to maximize his utility. The utility maximization problem can now be easily solved.

Lemma 2.1 Let $b_{i}=\gamma_{i}^{-1}$. The solution to the utility maximization problem for agent $i$,

$$
\max \left\{E\left[\sum_{t=0}^{\infty} \delta_{i}^{t} \frac{x_{i t}^{1-\gamma_{i}}-1}{1-\gamma_{i}}\right] \mid E\left[\sum_{t=0}^{\infty} x_{i t} M_{t}\right]=\eta_{i} E\left[\sum_{t=0}^{\infty} W_{t} M_{t}\right]\right\}
$$

\footnotetext{
${ }^{2}$ The reciprocal of relative risk aversion, $b_{i}=1 / \gamma_{i}$, is called cautiousness.
} 
is given by $x_{i t}=M_{t}^{-b_{i}} \delta_{i}^{t b_{i}} x_{i 0}$ for all $t \geq 1$ and

$$
x_{i 0}=\eta_{i} \frac{\sum_{t=0}^{\infty} E\left[W_{t} M_{t}\right]}{\sum_{t=0}^{\infty} \delta_{i}^{t b_{i}} E\left[M_{t}^{1-b_{i}}\right]} .
$$

\section{Market equilibrium}

If the payoffs of all assets are linearly independent, standard arguments (see, e.g., $[8,13])$ imply that the equilibrium market clearing for all assets is equivalent to the market clearing for the consumption good. Thus, our equilibrium can be characterized as an Arrow-Debreu equilibrium.

Definition 3.1 A positive state price density process $\mathbf{M}:=\left(M_{t}, t \geq 0\right)$ is an ArrowDebreu equilibrium if

$$
\sum_{i \in N} x_{i t}=W_{t}
$$

for all $t \geq 0$. That is,

$$
\sum_{i \in N} \delta_{i}^{t b_{i}} M_{t}^{-b_{i}} x_{i 0}=W_{t}
$$

for all $t \geq 1$ (recall that $b_{i}=\gamma_{i}^{-1}$ ). Market clearing at time zero follows from Walras' law.

Existence of an equilibrium for infinite horizon economies is a nontrivial problem. In [16] we prove the following:

Theorem 3.2 Under the assumptions above, an equilibrium exists if and only if

$$
\delta_{i} E\left[X_{1}^{1-\gamma_{i}}\right]<1
$$

for all $i=1, \ldots, n$. This condition is also necessary and sufficient for the finiteness of the Lucas tree equity price.

Therefore, everywhere in the sequel we make the following:

Assumption 3.3 Inequality (3.2) is fulfilled for any $i=1, \ldots, n$.

In general, if the state space and horizon are finite, the existence follows from standard results (see, e.g., [6]). Then we could simply view the infinite horizon yields as limits of finite horizon yields as the horizon goes to infinity. But viewing the yields directly as prices in infinite horizon economies is, of course, more convenient. For this reason, we present a sketch of the proof of Theorem 3.2 in the Appendix. 
Nonuniqueness of equilibria Note that the equilibrium in our economy is not necessarily unique (see [16] for concrete examples of nonuniqueness). But these multiple equilibria only differ from each other via the initial consumptions $x_{i 0}$. Our results are universal because they are independent of the initial consumptions $x_{i 0}$ and, consequently, are independent of a particular equilibrium.

\section{The aggregator function and its properties}

The key ingredient of the proofs is the aggregator function constructed in the following:

Proposition 4.1 In equilibrium, the $\tau$-period stochastic discount factor is given by

$$
\frac{M_{t+\tau}}{M_{t}}=F_{t}\left(\delta_{1}^{\tau}\left(\frac{W_{t+\tau}}{W_{t}}\right)^{-\gamma_{1}}, \ldots, \delta_{n}^{\tau}\left(\frac{W_{t+\tau}}{W_{t}}\right)^{-\gamma_{n}}\right),
$$

where $F_{t}=F_{t}\left(y_{1}, \ldots, y_{n}\right)$ is the unique solution to

$$
\sum_{i=1}^{n} F_{t}^{-b_{i}} y_{i}^{b_{i}}\left(x_{i t} W_{t}^{-1}\right)=1
$$

Note that the weights $x_{i t} W_{t}^{-1}$ sum to one.

Proof Dividing the equilibrium equations (3.1) at time $t+\tau$ by $W_{t+\tau}$, we get

$$
\begin{aligned}
1 & =\sum_{i=1}^{n} e^{-\rho_{i}(t+\tau) b_{i}} M_{t+\tau}^{-b_{i}} x_{i 0} W_{t+\tau}^{-1} \\
& =\sum_{i=1}^{n} e^{-\rho_{i} \tau b_{i}}\left(\frac{M_{t+\tau}}{M_{t}}\right)^{-b_{i}} M_{t}^{-b_{i}} e^{-\rho_{i} t b_{i}} x_{i 0} W_{t}^{-1} W_{t} W_{t+\tau}^{-1} \\
& =\sum_{i=1}^{n} e^{-\rho_{i} \tau b_{i}}\left(\frac{M_{t+\tau}}{M_{t}}\right)^{-b_{i}}\left(x_{i t} W_{t}^{-1}\right)\left(\left(\frac{W_{t+\tau}}{W_{t}}\right)^{-\gamma_{i}}\right)^{b_{i}} \\
& =\sum_{i=1}^{n}\left(\frac{M_{t+\tau}}{M_{t}}\right)^{-b_{i}}\left(e^{-\rho_{i} \tau}\left(\frac{W_{t+\tau}}{W_{t}}\right)^{-\gamma_{i}}\right)^{b_{i}}\left(x_{i t} W_{t}^{-1}\right),
\end{aligned}
$$

and the claim immediately follows.

Proposition 4.1 allows us to formulate the most important properties of the state price density process in terms of the aggregator function. In Malamud [15], we use the aggregator function to prove sharp estimates for asset prices.

We will need the following important lemma. 
Lemma 4.2 The function $F_{t}$ satisfies

$$
\begin{aligned}
\max _{i}\left(y_{i}\left(x_{i t} W_{t}^{-1}\right)^{\gamma_{i}}\right) & \leq F_{t}\left(y_{1}, \ldots, y_{n}\right) \\
& \leq\left(\sum_{i \in N} y_{i}^{\gamma^{-1}}\left(x_{i t} W_{t}^{-1}\right)^{\gamma^{-1} \gamma_{i}}\right)^{\gamma} \leq n^{\gamma-1} \sum_{i \in N} y_{i},
\end{aligned}
$$

where $\gamma$ is any number satisfying the inequality $\gamma^{-1} \leq \min \left\{\min _{i} b_{i}, 1\right\}$.

Proof The first inequality follows from

$$
F_{t}^{-b_{j}} y_{j}^{b_{j}} x_{j t} W_{t}^{-1} \leq \sum_{i=1}^{n} F_{t}^{-b_{i}} y_{i}^{b_{i}}\left(x_{i t} W_{t}^{-1}\right)=1
$$

for any $j \in N$. Suppose now that

$$
F_{t}\left(y_{1}, \ldots, y_{n}\right)>\left(\sum_{i \in N} y_{i}^{\gamma^{-1}}\left(x_{i t} W_{t}^{-1}\right)^{\gamma^{-1} \gamma_{i}}\right)^{\gamma} .
$$

Then

$$
\begin{aligned}
1 & =\sum_{i=1}^{n} F_{t}^{-b_{i}} y_{i}^{b_{i}}\left(x_{i t} W_{t}^{-1}\right)<\sum_{i=1}^{n}\left(\frac{y_{i}^{\gamma^{-1}}\left(x_{i t} W_{t}^{-1}\right)^{\gamma^{-1} \gamma_{i}}}{\sum_{i \in N} y_{i}^{\gamma^{-1}}\left(x_{i t} W_{t}^{-1}\right)^{\gamma^{-1} \gamma_{i}}}\right)^{b_{i} \gamma} \\
& \leq \sum_{i=1}^{n} \frac{y_{i}^{\gamma^{-1}}\left(x_{i t} W_{t}^{-1}\right)^{\gamma^{-1} \gamma_{i}}}{\sum_{i \in N} y_{i}^{\gamma^{-1}}\left(x_{i t} W_{t}^{-1}\right)^{\gamma^{-1} \gamma_{i}}}=1
\end{aligned}
$$

which is a contradiction. The last inequality follows from Jensen's inequality and convexity of $x^{\gamma}$.

\section{The long run yield of a zero coupon bond and long run forward rates}

Recall that the aggregate endowment $\left(W_{t}\right)$ is assumed to be a geometric random walk,

$$
W_{t}=X_{1} \cdots X_{t}
$$

with i.i.d. $X_{t}$. In a homogeneous economy populated by identical agents with risk aversion $\gamma$ and discount factors $\delta$, bond prices are constant and are given by

$$
B^{\mathrm{F}}(t, t+\tau)(\delta, \gamma)=\delta^{\tau}\left(E\left[X_{1}^{-\gamma}\right]\right)^{\tau},
$$

and the yield of the zero coupon bond is, by definition,

$$
-\tau^{-1} \log B^{\mathrm{F}}(t, t+\tau)(\delta, \gamma)=-\log \left(\delta E\left[X_{1}^{-\gamma}\right]\right) .
$$


When agents are heterogeneous, bond prices cannot be calculated explicitly anymore, but their asymptotic behavior can be studied in detail. The following result is a substantial extension of Theorem 4 in Wang [22] for the general class of heterogeneous CRRA economies.

\section{Theorem 5.1 We have}

$$
\lim _{\tau \rightarrow \infty} \tau^{-1} \log B^{\mathrm{F}}(t, t+\tau)=\max _{i} \log \left(\delta_{i} E\left[X_{1}^{-\gamma_{i}}\right]\right) .
$$

Remark 5.2 Wang [22] considered an economy with two agents having identical discount factors and risk aversions 1 and $1 / 2$ and proved (5.1) in this very special case.

The proof of Theorem 5.1 is based on the following simple lemma.

Lemma 5.3 Let $\rho_{i} \in \mathbb{R}, i=1, \ldots, m$. If $a_{i}(\tau)=e^{\tau \rho_{i}+O(1)}$ for all $\tau>0$, then

$$
\lim _{\tau \rightarrow \infty} \tau^{-1} \log \sum_{i=1}^{m} a_{i}(\tau)=\max _{i} \rho_{i} .
$$

Proof of Theorem 5.1 By Lemma 4.2 and Proposition 4.1,

$$
\begin{aligned}
\delta_{j}^{\tau} & \left(E\left[X_{1}^{-\gamma_{j}}\right]\right)^{\tau}\left(x_{j t} W_{t}^{-1}\right)^{\gamma_{j}} \\
& =\delta_{j}^{\tau} E_{t}\left[\left(W_{t+\tau} / W_{t}\right)^{-\gamma_{j}}\right]\left(x_{j t} W_{t}^{-1}\right)^{\gamma_{j}} \\
& \leq E_{t}\left[\frac{M_{t+\tau}}{M_{t}}\right]=B^{\mathrm{F}}(t, t+\tau) \leq n^{\gamma-1} \sum_{i \in N} \delta_{i}^{\tau} E_{t}\left[\left(W_{t+\tau} / W_{t}\right)^{-\gamma_{i}}\right] \\
& =n^{\gamma-1} \sum_{i \in N} \delta_{i}^{\tau}\left(E\left[X_{1}^{-\gamma_{i}}\right]\right)^{\tau}
\end{aligned}
$$

for any $j \in N$. The required assertion immediately follows.

A much more subtle question is to understand the behavior of the forward rates

$$
f_{t}(\tau)=\frac{B^{\mathrm{F}}(t, t+\tau)}{B^{\mathrm{F}}(t, t+\tau+1)} .
$$

Note that the bond yields satisfy

$$
-\tau^{-1} \log B^{\mathrm{F}}(t, t+\tau)=\tau^{-1} \sum_{\theta=1}^{\tau} \log f_{t}(\theta),
$$

and, consequently, if the forward rates converge to a limit, so do the bond yields (see also [10] and [12]), but the converse is generally not true.

Surprisingly, this is true in our class of economies under a simple genericity assumption. 
Assumption 5.4 There exists a unique agent $b \in N$ such that

$$
\delta_{b} E\left[X_{1}^{-\gamma_{b}}\right]=\max _{i} \delta_{i} E\left[X_{1}^{-\gamma_{i}}\right] .
$$

Obviously, Assumption 5.4 holds for generic economies (the set of such economies is a complement of a finite number of smooth hypersurfaces).

Theorem 5.5 Let Assumption 5.4 be fulfilled. Then

$$
\lim _{\tau \rightarrow \infty} f_{t}(\tau)=\left(\delta_{b} E\left[X_{1}^{-\gamma_{b}}\right]\right)^{-1} .
$$

We will need the following auxiliary lemma.

Lemma 5.6 Let $\gamma \geq 1$. Then the function $f: R_{+}^{n} \rightarrow \mathbb{R}$ defined by

$$
f\left(x_{1}, \ldots, x_{n}\right)=\left(\sum_{i=1}^{n} x_{i}^{1 / \gamma}\right)^{\gamma}
$$

is concave.

Proof The Hessian $H(f)$ of the function $f$ is given by

$$
\begin{aligned}
H(f)= & \left(\partial^{2} f / \partial x_{i} \partial x_{j}\right)_{i, j=1}^{n} \\
= & \left(1-\gamma^{-1}\right)\left(\sum_{i=1}^{n} x_{i}^{1 / \gamma}\right)^{\gamma-2}\left(\left(x_{i}^{1 / \gamma-1} x_{j}^{1 / \gamma-1}\right)_{i, j=1}^{n}\right. \\
& \left.-\left(\sum_{i=1}^{n} x_{i}^{1 / \gamma}\right) \operatorname{diag}\left(x_{i}^{1 / \gamma-2}\right)_{i=1}^{n}\right) \\
= & \left(1-\gamma^{-1}\right) f^{1-2 / \gamma} \operatorname{diag}\left(x_{i}^{\gamma^{-1} / 2-1}\right)_{i=1}^{n} \\
& \times\left(\left(x_{i}^{\gamma^{-1} / 2} x_{j}^{\gamma^{-1} / 2}\right)_{i, j=1}^{n}-f^{1 / \gamma} I\right) \operatorname{diag}\left(x_{i}^{\gamma^{-1} / 2-1}\right)_{i=1}^{n} .
\end{aligned}
$$

Here, $I$ is the identity matrix. The matrix

$$
A=\left(x_{i}^{\gamma^{-1} / 2} x_{j}^{\gamma^{-1} / 2}\right)_{i, j=1}^{n}
$$

equals $f^{1 / \gamma}$ times the orthogonal projection onto the vector $\left(x_{i}^{\gamma^{-1} / 2}\right)_{i=1}^{n}$. Thus, $\|A\|=f^{1 / \gamma}$, and the matrix

$$
\left(x_{i}^{\gamma^{-1} / 2} x_{j}^{\gamma^{-1} / 2}\right)_{i, j=1}^{n}-f^{1 / \gamma} I
$$

is negative definite. Therefore, $H(f)$ is also negative definite.

The Jensen inequality immediately yields the following: 
Lemma 5.7 Let $\gamma \in \mathbb{N}$ be a natural number and $X_{1}, \ldots, X_{n}$ nonnegative random variables. Then

$$
E\left[\left(X_{1}^{1 / \gamma}+\cdots+X_{n}^{1 / \gamma}\right)^{\gamma}\right] \leq\left(E\left[X_{1}\right]^{1 / \gamma}+\cdots+E\left[X_{n}\right]^{1 / \gamma}\right)^{\gamma}
$$

We are now ready to prove the theorem.

Proof of Theorem 5.5 Let $b$ be the (by assumption unique) agent from Assumption 5.4 and

$$
\alpha_{j}=x_{j t} W_{t}^{-1}
$$

for all $j \in N$. Then, applying Lemma 5.7 to the random variables

$$
X_{j}=\delta_{j}^{\tau}\left(\frac{W_{t+\tau}}{W_{t}}\right)^{-\gamma_{j}} \alpha_{j}^{\gamma_{j}}
$$

and using Proposition 4.1 and Lemma 4.2, we arrive at the inequality

$$
\frac{E_{t}\left[M_{t+\tau}\right]}{M_{t}} \leq\left(\sum_{j=1}^{n}\left(\left(\delta_{j} E\left[X_{1}^{-\gamma_{j}}\right]\right)^{\tau} \alpha_{j}^{\gamma_{j}}\right)^{1 / \gamma}\right)^{\gamma}
$$

for some $\gamma>1$. Denote

$$
\kappa:=\frac{\max _{i \neq b}\left(\delta_{i} E\left[X_{1}^{-\gamma_{i}}\right]\right)}{\delta_{b} E\left[X_{1}^{-\gamma_{b}}\right]} .
$$

By Assumption 5.4, $\kappa<1$. Applying Lemma 4.2 and using (5.3), we get

$$
\left(\delta_{b} E\left[X_{1}^{-\gamma_{b}}\right]\right)^{\tau} \alpha_{b} \leq B^{\mathrm{F}}(t, t+\tau) \leq\left(\delta_{b} E\left[X_{1}^{-\gamma_{b}}\right]\right)^{\tau} \alpha_{b}\left(1+K \kappa^{\tau \gamma^{-1}}\right)^{\gamma}
$$

where

$$
K=\sum_{j \neq b}\left(\alpha_{j}^{\gamma_{j}} \alpha_{b}^{-\gamma_{b}}\right)^{1 / \gamma}
$$

Consequently,

$$
B^{\mathrm{F}}(t, t+\tau)=\left(\delta_{b} E\left[X_{1}^{-\gamma_{b}}\right]\right)^{\tau} \alpha_{b}(1+o(1)),
$$

and the forward rates satisfy

$$
\frac{B^{\mathrm{F}}(t, t+\tau)}{B^{\mathrm{F}}(t, t+\tau+1)}=\left(\delta_{b} E\left[X_{1}^{-\gamma_{b}}\right]\right)^{-1}(1+o(1)),
$$

which gives the assertion. 


\section{The yield of a long maturity European call option}

Recall that the Lucas tree equity is the asset whose dividend process coincides with the aggregate endowment, and its price $P_{t}^{\mathrm{W}}$ is given by

$$
P_{t}^{\mathbf{W}}=E_{t}\left[\sum_{\tau=1}^{\infty} \frac{M_{t+\tau}}{M_{t}} W_{t+\tau}\right] .
$$

Note that in a homogeneous economy with parameters $(\delta, \gamma)$, the equity price is proportional to the dividend and is given by

$$
P_{t}^{\mathbf{W}}(\delta, \gamma)=W_{t} \frac{\delta E\left[X_{1}^{1-\gamma}\right]}{1-\delta E\left[X^{1-\gamma}\right]} .
$$

In general, Proposition 4.1 and Lemma 4.2 together yield the following:

\section{Lemma 6.1}

$$
C_{1} W_{t} \leq P_{t}^{\mathbf{W}} \leq C_{2} W_{t}
$$

for some constants $C_{2}>C_{1}>0$.

Proof It follows directly from Proposition 4.1 and Lemma 4.2 that the constants

$$
C_{1}=\frac{\delta_{1} E\left[X_{1}^{1-\gamma_{1}}\right]}{1-\delta_{1} E\left[X_{1}^{1-\gamma_{1}}\right]}
$$

and

$$
C_{2}=n^{\gamma-1} \sum_{j \in N} \frac{\delta_{j} E\left[X_{1}^{1-\gamma_{j}}\right]}{1-\delta_{1} j, E\left[X_{1}^{1-\gamma_{j}}\right]}
$$

satisfy the required estimates.

By absence of arbitrage, the price $\operatorname{Call}_{t}(K, t+\tau)$ at time $t$ of a European call option with strike $K$ and maturity $t+\tau$ is given by

$$
\operatorname{Call}_{t}(K, t+\tau)=E_{t}\left[\frac{M_{t+\tau}}{M_{t}}\left(P_{t+\tau}^{\mathbf{W}}-K\right)^{+}\right] .
$$

The payoff of the option at maturity is, by definition, $\left(P_{t+\tau}^{\mathbf{W}}-K\right)^{+}$, and, therefore, the log expected per-period return on holding the option up to maturity (i.e., the option yield) is given by

$$
\tau^{-1} \log \frac{E_{t}\left[\left(P_{t+\tau}^{\mathbf{W}}-K\right)^{+}\right]}{E_{t}\left[\frac{M_{t+\tau}}{M_{t}}\left(P_{t+\tau}^{\mathbf{W}}-K\right)^{+}\right]} .
$$

In this section, we study the asymptotic behavior of the option yield as its maturity tends to infinity. We will need several definitions. 
Assumption 6.2 We assume that the jump $X_{1}$ of the aggregate endowment satisfies the Donsker-Varadhan condition

$$
E\left[X_{1}^{\alpha}\right]<\infty
$$

for all $\alpha \in \mathbb{R}$. This condition guarantees that the Cramér large deviation result holds. See, e.g., [7], p. 6.

Definition 6.3 For a random variable $X \geq 0$, let

$$
Z(\beta, X)=E\left[X^{\beta}\right]
$$

and

$$
S(x, X)=\sup _{\beta \in \mathbb{R}}(x \beta-\ln Z(\beta, X)) .
$$

The function $S(x, X)$ is the Legendre transform of the moment-generating function $Z$.

Lemma 6.4 $S(x, X)$ is a strictly convex function of

$$
x \in(\text { essinf } \log X, \text { esssup } \log X)
$$

with

$$
\min _{x} S(x, X)=S(E[\log X], X)=0 .
$$

Consequently, $S(x, X)>0$ for all $x \neq E[\log X]$.

Lemma 6.4 means that $S(x, X)$ measures the deviation of $x$ from the mean $E[\log X]$.

If $H=\log X$ is a binomial variable taking values $h_{1}<h_{2}$ with probabilities $\mathrm{p}_{1}$ and $\mathrm{p}_{2}=\mathrm{p}$, then

$$
S(x, X)=-I_{y}(\mathrm{p}),
$$

where

$$
y=\mathrm{p}+\frac{x-E[H]}{h_{2}-h_{1}},
$$

and $I_{y}(\mathrm{p})$ is the relative entropy function given by

$$
I_{y}(\mathrm{p})=y \log \left(\frac{\mathrm{p}}{y}\right)+(1-y) \log \left(\frac{1-\mathrm{p}}{1-y}\right) .
$$

Cramér's large deviations theorem states that $S(x, X)$ is the exact rate at which $\log X$ deviates from its mean. Namely, the following is true. 
Theorem 6.5 (Cramér's large deviations theorem) As $\tau \rightarrow \infty$, we have

$$
P\left[W_{\tau} \geq e^{\tau x}\right]=e^{-\tau\left(S\left(x, X_{1}\right)+o(1)\right)}
$$

for any $x$ satisfying esssup $X_{1}>x \geq E\left[\log X_{1}\right]$. In particular, if $E\left[\log X_{1}\right]<0<$ esssup $X_{1}$, then, for any positive constant $K>0$,

$$
P\left[W_{\tau} \geq K\right]=e^{-\tau\left(S\left(0, X_{1}\right)+o(1)\right)} \quad \text { as } \tau \rightarrow \infty .
$$

See, e.g., [7], p. 6 for a proof. We will need modifications of the large deviations theorem under an equivalent change of measure.

Definition 6.6 On each sigma-algebra $\mathcal{F}_{t}$, define the equivalent probability measure

$$
d P_{t}^{\gamma}=\frac{W_{\tau}^{-\gamma}}{E\left[W_{\tau}^{-\gamma}\right]} d P
$$

It is easy to see that the family of measures $P_{t}^{\gamma}$ is consistent (because $\left(W_{t}\right)$ is a geometric random walk), and therefore, by the Kolmogorov extension theorem, there exists a measure $P^{\gamma}$ whose restriction on each finite horizon algebra $\mathcal{F}_{t}$ coincides with $P_{t}^{\gamma}$. The mathematical expectation with respect to $P^{\gamma}$ is denoted by $E^{\gamma}$.

It is easy to see that $\mathbf{W}=\left(W_{t}\right)$ is also a random walk under this modified probability measure $P^{\gamma}$, that the Donsker-Varadhan condition is also fulfilled, and therefore the large deviations theorem also holds. We denote by

$$
Z^{\gamma}(\beta, X)=E^{\gamma}\left[X^{\beta}\right]
$$

and

$$
S^{\gamma}(x, X)=\sup _{\beta \in \mathbb{R}}\left(x \beta-\ln Z^{\gamma}(\beta, X)\right)
$$

the corresponding Cramér function. Then the large deviations theorem takes the form

$$
P^{\gamma}\left[W_{\tau} \geq e^{\tau x}\right]=e^{-\tau\left(S^{\gamma}\left(x, X_{1}\right)+o(1)\right)} \quad \text { as } \tau \rightarrow \infty .
$$

We will also make the following economically natural assumption.

Assumption 6.7 $P\left[X_{1}>1\right] \cdot P\left[X_{1}<1\right]>0$. That is,

$$
0 \in\left(\text { essinf } \log X_{1}, \text { esssup } \log X_{1}\right)
$$

Assumption 6.7 means that both booms (growth) and recessions happen with positive probability.

Lemma 6.8 The function $f(\gamma)=E\left[X_{1}^{-\gamma}\right]$ is strictly convex in $\gamma$. Under Assumption 6.7,

$$
\lim _{\gamma \rightarrow+\infty} f(\gamma)=\lim _{\gamma \rightarrow-\infty} f(\gamma)=+\infty
$$


and $f(\gamma)$ has a unique global minimum $\mathfrak{G}$ satisfying

$$
E\left[X_{1}^{-\mathfrak{G}} \log X_{1}\right]=0 .
$$

Furthermore, $\mathfrak{G}>0$ if and only if

$$
E\left[\log X_{1}\right]>0
$$

Proof All claims are immediate consequences of the definitions. The positivity of $\mathfrak{G}$ follows because $f^{\prime}(0)=-E\left[\log X_{1}\right]$.

The "critical" risk aversion $\mathfrak{G}$ plays a very important role in our analysis. The following result will be crucial for our considerations.

Lemma 6.9 The function

$$
g(\gamma)=-\frac{\partial}{\partial \gamma} \log f=E^{\gamma}\left[\log X_{1}\right]=\frac{E\left[X_{1}^{-\gamma} \log X_{1}\right]}{E\left[X_{1}^{-\gamma}\right]}
$$

is decreasing in $\gamma$ and satisfies

$$
g(\mathfrak{G})=0 .
$$

Consequently,

$$
S^{\gamma}\left(0, X_{1}\right)=-\log \frac{E\left[X_{1}^{-\mathfrak{G}}\right]}{E\left[X_{1}^{-\gamma}\right]} .
$$

We will need the following well-known correlation inequality.

Lemma 6.10 If $h_{1}(x)$ and $h_{2}(x)$ are increasing, then

$$
E\left[h_{1}(X) h_{2}(X)\right] \geq E\left[h_{1}(X)\right] E\left[h_{2}(X)\right]
$$

for any random variable $X$.

Proof of Lemma 6.9 Using Lemma 6.10, we get

$$
E^{\gamma_{1}}\left[\log X_{1}\right]=\frac{E^{\gamma_{2}}\left[X_{1}^{\gamma_{2}-\gamma_{1}} \log X_{1}\right]}{E^{\gamma_{2}}\left[X_{1}^{\gamma_{2}-\gamma_{1}}\right]} \geq E^{\gamma_{2}}\left[\log X_{1}\right]
$$

for any $\gamma_{2} \geq \gamma_{1}$, and the required monotonicity follows. Now,

$$
S^{\gamma}\left(0, X_{1}\right)=-\log \frac{E\left[X^{\beta-\gamma}\right]}{E\left[X^{-\gamma}\right]},
$$

where $\beta$ satisfies the first-order condition

$$
E\left[X_{1}^{\beta-\gamma} \log X_{1}\right]=0 .
$$

That is, $\beta=\gamma-\mathfrak{G}$, and the claim follows. 


\section{Theorem 6.11 Let}

$$
\mathcal{O}(\delta, \gamma)= \begin{cases}\log \left(\delta E\left[X_{1}^{1-\gamma}\right]\right), & \mathfrak{G} \geq \gamma-1 \\ \log \left(\delta E\left[X_{1}^{-\mathfrak{G}}\right]\right), & \mathfrak{G} \leq \gamma-1\end{cases}
$$

Then

$$
\lim _{\tau \rightarrow \infty} \tau^{-1} \log \frac{E_{t}\left[\left(P_{\tau}^{\mathbf{W}}-K\right)^{+}\right]}{E_{t}\left[M_{\tau}\left(P_{\tau}^{\mathbf{W}}-K\right)^{+}\right]}=\mathcal{O}(1,0)-\max _{i} \mathcal{O}\left(\delta_{i}, \gamma_{i}\right) .
$$

Proof We consider only the case $t=0$. The case $t>0$ is completely analogous. We will first show that

$$
\lim _{\tau \rightarrow \infty} \tau^{-1} \log E\left[M_{\tau}\left(P_{\tau}^{\mathbf{W}}-K\right)^{+}\right]=\max _{i} \mathcal{O}\left(\delta_{i}, \gamma_{i}\right)
$$

In fact, we will prove that

$$
\lim _{\tau \rightarrow \infty} \tau^{-1} \log E\left[M_{\tau}\left(C W_{\tau}-K\right)^{+}\right]=\max _{i} \mathcal{O}\left(\delta_{i}, \gamma_{i}\right)
$$

for any constant $C>0$. Then the required assertion will follow from Lemma 6.1. Proposition 4.1 and Lemma 4.2 together yield that

$$
n^{-1}\left(\min _{j}\left(x_{j 0}\right)^{\gamma_{j}}\right) \sum_{i \in N} \delta_{i}^{\tau} W_{\tau}^{-\gamma_{i}} \leq M_{\tau} \leq n^{\gamma-1} \sum_{i \in N} \delta_{i}^{\tau} W_{\tau}^{-\gamma_{i}} .
$$

Consequently, it suffices to prove that

$$
\lim _{\tau \rightarrow \infty} \tau^{-1} \log E\left[\sum_{i \in N} \delta_{i}^{\tau} W_{\tau}^{-\gamma_{i}}\left(W_{\tau}-K\right)^{+}\right]=\max _{i} \mathcal{O}\left(\delta_{i}, \gamma_{i}\right),
$$

since the limit is independent of $C$ and $K$. Denote

$$
\pi(K, \tau, \gamma)=P^{\gamma}\left[W_{\tau}>K\right]=\left(E\left[X_{1}^{-\gamma}\right]\right)^{-\tau} E\left[W_{\tau}^{-\gamma}, W_{\tau}>K\right] .
$$

We have

$$
\begin{aligned}
E\left[\delta_{i}^{\tau} W_{\tau}^{-\gamma_{i}}\left(W_{\tau}-K\right)^{+}\right] & =E\left[\delta_{i}^{\tau} W_{\tau}^{1-\gamma_{i}}-K \delta_{i}^{\tau} W_{\tau}^{-\gamma_{i}}, W_{\tau}>K\right] \\
& =f\left(\delta_{i}, \gamma_{i}-1\right)^{\tau} \pi\left(K, \tau, \gamma_{i}-1\right)-K f\left(\delta_{i}, \gamma_{i}\right)^{\tau} \pi\left(K, \tau, \gamma_{i}\right) \\
& \leq f\left(\delta_{i}, \gamma_{i}-1\right)^{\tau} \pi\left(K, \tau, \gamma_{i}-1\right)
\end{aligned}
$$

and

$$
\begin{aligned}
E\left[\delta_{i}^{\tau} W_{\tau}^{-\gamma_{i}}\left(W_{\tau}-K\right)^{+}\right] & =E\left[\delta_{i}^{\tau} W_{\tau}^{1-\gamma_{i}}-K \delta_{i}^{\tau} W_{\tau}^{-\gamma_{i}}, W_{\tau}>K\right] \\
& \geq E\left[\delta_{i}^{\tau} W_{\tau}^{1-\gamma_{i}}-K \delta_{i}^{\tau} W_{\tau}^{-\gamma_{i}}, W_{\tau}>2 K\right] \\
& \geq \frac{1}{2} E\left[\delta_{i}^{\tau} W_{\tau}^{1-\gamma_{i}}, W_{\tau}>2 K\right] \\
& =\frac{1}{2} f\left(\delta_{i}, \gamma_{i}-1\right)^{\tau} \pi\left(2 K, \tau, \gamma_{i}-1\right) .
\end{aligned}
$$


By Cramér's large deviations theorem, the asymptotic behavior of $\pi(K, \tau, \gamma)$ is independent of $K$, and therefore it suffices to understand the asymptotic behavior of the right-hand side of (6.5). We will consider two cases.

(1) $\gamma_{i} \geq \mathfrak{G}+1$. In this case, Lemma 6.9 implies that

$$
E^{\gamma_{i}-1}\left[\log X_{1}\right] \leq 0
$$

Therefore, Cramér's large deviations theorem and Lemma 6.9 together yield that

$$
\pi\left(K, \tau, \gamma_{i}-1\right)=e^{-\tau\left(S^{\gamma_{i}-1}\left(0, X_{1}\right)+o(1)\right)}=\left(\frac{E\left[X_{1}^{-\mathfrak{G}}\right]}{E\left[X_{1}^{1-\gamma_{i}}\right]}\right)^{\tau} e^{-\tau o(1)}
$$

and, consequently,

$$
f\left(\delta_{i}, \gamma_{i}-1\right)^{\tau} \pi\left(K, \tau, \gamma_{i}-1\right)-K f\left(\delta_{i}, \gamma_{i}\right)^{\tau} \pi\left(K, \tau, \gamma_{i}\right)=f\left(\delta_{i}, \mathfrak{G}\right)^{\tau} e^{\tau o(1)} .
$$

(2) $\gamma_{i}<\mathfrak{G}+1$. Then

$$
E^{\gamma_{i}-1}\left[\log X_{1}\right]>0
$$

and therefore

$$
\pi\left(K, \tau, \gamma_{i}\right)=1-e^{-\tau(a+o(1))}
$$

for a positive constant $a>0$.

Thus, we have proved that

$$
E\left[\delta_{i}^{\tau} W_{\tau}^{-\gamma_{i}}\left(W_{\tau}-K\right)^{+}\right]=\mathcal{O}\left(\delta_{i}, \gamma_{i}\right)^{\tau} e^{\tau o(1)}
$$

as $\tau \rightarrow \infty$, and (6.4) immediately follows. Finally, by the same arguments,

$$
\lim _{\tau \rightarrow \infty} \tau^{-1} \log E\left[\left(P_{\tau}^{\mathbf{W}}-K\right)^{+}\right]=\log \mathcal{O}(1,0)= \begin{cases}\log E\left[X_{1}\right], & \mathfrak{G} \geq-1, \\ \log E\left[X_{1}^{-\mathfrak{G}}\right], & \mathfrak{G} \leq-1 .\end{cases}
$$

Remark 6.12 Theorem 6.11 generates many theoretical predictions that should not be difficult to test with real data.

The first amazing consequence of Theorem 6.11 is that the option yield is independent of the risk aversions $\gamma_{i}$ if $\gamma_{i} \geq \mathfrak{G}+1$.

The second, even more interesting consequence is that the option yield is always greater than or equal to the corresponding equity return. Namely, in a homogeneous economy with parameters $(\delta, \gamma)$, the equity return is given by

$$
r_{t+1}^{\mathbf{W}}=\frac{P_{t+1}^{\mathbf{W}}(\delta, \gamma)+W_{t+1}}{P_{t}^{\mathbf{W}}(\delta, \gamma)}=\frac{W_{t+1}}{W_{t}} \frac{1}{f(\delta, \gamma-1)},
$$

and consequently, the expected cumulative return on constantly reinvesting all the money in equity is given by

$$
E\left[r_{1}^{\mathbf{W}} r_{2}^{\mathbf{W}} \cdots r_{t}^{\mathbf{W}}\right]=\left(\frac{E\left[X_{1}\right]}{f(\delta, \gamma-1)}\right)^{\tau}
$$


Thus, the per-period return on holding equity is given by

$$
\frac{E\left[X_{1}\right]}{f(\delta, \gamma-1)} \text {. }
$$

Now, if the dividends $\mathbf{W}$ are growing on average, i.e., $E\left[\log X_{1}\right]>0$, then the perperiod return on holding a long maturity call option is by Theorem 6.11 given by

$$
\frac{E\left[X_{1}\right]}{f(\delta, \min \{\gamma-1, \mathfrak{G}\})} \geq \frac{E\left[X_{1}\right]}{f(\delta, \gamma-1)},
$$

where the last inequality follows from Lemma 6.8. That is, in the long run, it is more profitable to hold a long maturity option than the equity itself.

This is a very important theoretical prediction. It would be very interesting to compare yields on long maturity call options with long run cumulative equity returns and check the above theoretical prediction. In particular, it would allow us to get some idea about the size of risk aversion $\gamma$ and to find out whether $\gamma \geq(\leq) \mathfrak{G}$.

\section{Appendix: A sketch of the proof for Theorem 3.2}

To prove the existence, we introduce the excess utility map (see, e.g., [6]). We introduce new parameters (social utility weights) $\lambda_{i}:=x_{i 0}^{\gamma_{i}}$ (note that we do not normalize the $\lambda_{i}$ to sum to 1$)$. Let $\mathbb{T}=\{1,2, \ldots\}$ and $e: \mathbb{R}_{++}^{n} \rightarrow \mathbb{R}^{n}$ be defined by

$$
e_{i}\left(\lambda_{1}, \ldots, \lambda_{n}\right):=\lambda_{i}^{-1}\left(\lambda_{i}^{b_{i}}\left(\sum_{t \in \mathbb{T}} \delta_{i}^{t b_{i}} E\left[G_{t}^{1-b_{i}}\right]\right)-\sum_{t \in \mathbb{T}} E\left[w_{i t} G_{t}\right]\right),
$$

where $G_{t}=G_{t}\left(\lambda_{1}, \ldots, \lambda_{n}, s\right)$ solves

$$
\sum_{i \in N} G_{t}^{-b_{i}} \delta_{i}^{t b_{i}} \lambda_{i}^{b_{i}}=W_{t}(s)
$$

(We do not use here the normalization $G_{0}=1$ (i.e., $M_{0}=1$ ), but $G_{0}$ fulfills the same kind of equation as $G_{t}$ for $t \geq 1$.)

Proposition 7.1 The state price densities $M_{t}, t \geq 1$, solve the equilibrium equations if and only if

$$
e\left(\lambda_{1}, \ldots, \lambda_{n}\right)=0
$$

We prove that $e$ has all the properties of an excess demand, and then the standard existence result from [17], p. 585, implies the existence.

Lemma 7.2 The excess utility map e satisfies:

1. $e$ is homogeneous of degree zero;

2. $\sum_{i \in N} \lambda_{i} e_{i}=0$; 
3. $e$ is continuous in $\mathbb{R}_{++}^{n}$;

4. $e_{i}$ is bounded from above for all $i$, and if $\lambda_{i} \rightarrow 0$, then $e_{i} \rightarrow-\infty$.

Properties 1 and 2 follow from the definition of $e$.

Property 3. We treat the two terms in (7.1) separately.

We now apply the trick of viewing a sequence of random variables as one variable, but on a larger probability space. Let $\Omega_{\infty}=\Omega \times \mathbf{T}$ be the union of an infinite number of identical copies of $\Omega$, and let $\nu_{\infty}$ be the infinite product measure on $\Omega_{\infty}$ coinciding with $\mu$ (the original probability measure on $\Omega$ ) on any copy of $\Omega$. Let, for $\Lambda:=\left(\lambda_{1}, \ldots, \lambda_{n}\right), \mathbf{G}(\Lambda)$ be the random variable on $\Omega_{\infty}$ equal to $G_{t}$ on the $t$-th copy. In just the same way, we define $\mathbf{W}=\left(W_{t}\right)_{t \geq 0}$ and $\mathbf{w}_{i}=\left(w_{i t}\right)$. Let also $\mathbf{D}_{i}=\left(\delta_{i}^{t}\right)_{t \in \mathbb{T}}$. Then we can rewrite (7.1) in the form

$$
e_{i}(\Lambda)=\lambda_{i}^{-1}\left(\lambda_{i}^{b_{i}} \int_{\Omega_{\infty}} \mathbf{D}_{i}^{b_{i}} \mathbf{G}^{1-b_{i}} d v_{\infty}-\int_{\Omega_{\infty}} \mathbf{w}_{i} \mathbf{G} d v_{\infty}\right) .
$$

Lemma 4.2 yields that

$$
\mathbf{G} \leq K \sum_{j} \lambda_{j} \mathbf{D}_{j} \mathbf{W}^{-\gamma_{j}}
$$

and therefore

$$
\mathbf{w}_{i} \cdot \mathbf{G} \leq \sum_{j} \lambda_{j} \mathbf{D}_{j} \mathbf{w}_{i} \mathbf{W}^{-\gamma_{j}} \leq \sum_{j \in N} \lambda_{j} \mathbf{D}_{j} \mathbf{W}^{1-\gamma_{j}} .
$$

In terms of $\Omega_{\infty}$, Assumption 3.2 means that the variable $\mathbf{D}_{j} \mathbf{W}^{1-\gamma_{j}}$ is $v_{\infty}$-integrable for any $j \in N$. From the definition of $G_{t}$ it follows that $G_{t}(\Lambda)$ is continuous in $\Lambda \in \mathbb{R}_{++}^{n}$ for any $t, s$. Estimate (7.4) and the dominated convergence theorem imply that we can pass to the limit under the integral $\int_{\Omega_{\infty}} \mathbf{w}_{i} \mathbf{G} d \nu_{\infty}$, and hence $\int_{\Omega_{\infty}} \mathbf{w}_{i} \mathbf{G} d \nu_{\infty}$ is continuous in $\Lambda$. To control the first term in (7.2), we note that for any compact set $X \subset \mathbb{R}_{++}^{n}$, there exists a constant $K$ (depending on $X$ ) such that

$$
\delta_{i}^{t b_{i}} G_{t}^{1-b_{i}}(\Lambda, s) \leq K \sum_{j \in N} \delta_{j}^{t} W_{t}^{1-\gamma_{j}}(s),
$$

that is,

$$
\mathbf{D}_{i}^{b_{i}} \mathbf{G}^{1-b_{i}} \leq K \sum_{j \in N} \mathbf{D}_{j} \mathbf{W}^{1-\gamma_{j}}
$$

Now, the dominated convergence theorem yields the required continuity.

Properties 4 and 5 . If $b_{i} \geq 1$, then

$$
\lambda_{i}^{b_{i}} \sum_{t \in \mathbb{T}} \delta_{i}^{t b_{i}} E\left[G_{t}^{1-b_{i}}\right] \leq \lambda_{i} \sum_{t \in \mathbb{T}} \delta_{i}^{t} E\left[W_{t}^{1-\gamma_{i}}\right]=\lambda_{i} K_{1} .
$$

From Lemma 4.2 it follows that

$$
\sum_{t \in \mathbb{T}} E\left[w_{i t} G_{t}\right] \geq K \sum_{j \in N} \lambda_{j} \sum_{t \in \mathbb{T}} \delta_{j}^{t} E\left[w_{j t} W_{t}^{-\gamma_{j}}\right] \geq K_{1} \sum_{j \in N} \lambda_{j}
$$


remains strictly positive. Thus we have

$$
e_{i} \leq K_{1}-K_{2} \lambda_{i}^{-1} \sum_{j=1}^{n} \lambda_{j}
$$

It follows that $e_{i}$ is uniformly bounded from above and tends to $-\infty$ as soon as not all $\lambda_{j}$ tend to zero.

If $b_{i}<1$, then

$$
\lambda_{i}^{b_{i}} \sum_{t \in \mathbb{T}} \delta_{i}^{t b_{i}} E\left[G_{t}^{1-b_{i}}\right] \leq K \lambda_{i}^{b_{i}} \sum_{j \in N} \lambda_{j}^{1-b_{i}}
$$

If $\lambda_{i}$ tends to zero and $\lambda_{j}$ stay bounded for $j \neq i$ and not all of them tend to zero, we have by (7.6) and (7.7) that

$$
e_{i} \leq \lambda_{i}^{-1}\left(K_{1} \lambda_{i}^{b_{i}} \sum_{j \in N} \lambda_{j}^{1-b_{i}}-K_{2} \sum_{j \in N} \lambda_{j}\right) .
$$

If $\lambda_{j}$ stay in a bounded region, $\lambda_{i} \rightarrow 0$, and $\lambda_{j} \neq 0$ at least for one $j$, then (7.8) implies

$$
e_{i} \leq-K_{3} \lambda_{i}^{-1} \rightarrow-\infty
$$

Finally, it is not difficult to see that

$$
\begin{aligned}
e_{i} & \leq \lambda_{i}^{-1} K_{1}\left(\lambda_{i}^{b_{i}} \sum_{j \in N} \lambda_{j}^{1-b_{i}}-\left(K_{2} K_{1}^{-1}\right) \sum_{j \in N} \lambda_{j}\right) \\
& \leq K_{1} \frac{L\left(K_{2} K_{1}^{-1}\right) \lambda_{i}}{\lambda_{i}}=K_{1} L\left(K_{2} K_{1}^{-1}\right) .
\end{aligned}
$$

\section{References}

1. Abel, A.B.: Asset pricing under habit formation and catching up with the Joneses. Am. Econ. Rev. (Papers and Proceedings) 80(2), 38-42 (1990)

2. Alvarez, F., Jermann, U.: Using asset prices to measure the persistence of the marginal utility of wealth. Econometrica 73, 1977-2016 (2005)

3. Benninga, S., Mayshar, J.: Heterogeneity and option pricing. Rev. Deriv. Res. 4, 7-27 (2000)

4. Constantinides, G.M.: Habit formation: a resolution of the equity premium puzzle. J. Political Econ. 98, 519-543 (1990)

5. Constantinides, G.M., Duffie, D.: Asset pricing with heterogeneous consumers. J. Political Econ. 104, 219-240 (1996)

6. Dana, R.-A.: Existence and uniqueness of equilibria when preferences are additively separable. Econometrica 61, 953-957 (1993)

7. Deuschel, J., Stroock, D.: Large Deviations. AMS Chelsea Publishing, New York (1989)

8. Duffie, D., Huang, C.-F.: Implementing Arrow-Debreu equilibria by continuous trading of few longlived securities. Econometrica 53, 1337-1356 (1985)

9. Dumas, B.: Two-person dynamic equilibrium in the capital market. Rev. Financial Stud. 2, 157-188 (1989) 
10. Dybvig, P.H., Ingersoll, J.E., Ross, S.A.: Long forward and zero-coupon rates can never fall. J. Bus. 69, 1-25 (1996)

11. Gollier, C., Zeckhauser, R.J.: Aggregation of heterogeneous time preferences. J. Political Econ. 113, 878-896 (2005)

12. Hubalek, F., Klein, I., Teichmann, J.: A general proof of the Dybvig-Ingersoll-Ross theorem: long forward rates can never fall. Math. Finance 12, 447-451 (2002)

13. Karatzas, I., Lehoczky, J.P., Shreve, S.E.: Existence and uniqueness of multi-agent equilibria in a stochastic, dynamic consumption/investment model. Math. Oper. Res. 15, 80-128 (1990)

14. Lengwiler, Y.: Heterogeneous patience and the term structure of real interest rates. Am. Econ. Rev. 95, 890-896 (2005)

15. Malamud, S.: Universal bounds for asset prices in heterogeneous economies. Working paper, SSRN (2007). http://ssrn.com/abstract=1002854

16. Malamud, S., Trubowitz, E.: Rational factor analysis, Working paper, SSRN (2006). http://ssrn.com/ abstract $=928132$

17. Mas-Colell, A., Whinston, M.D., Green, J.R.: Microeconomic Theory. Oxford University Press, Oxford (1995)

18. Ross, S.: Intertemporal asset pricing. In: Bhattachariya, S., Constantinides, G.M. (eds.) Theory of Valuation, Frontiers of Modern Financial Theory, vol. 1, pp. 85-96 (1989)

19. Rubinstein, M.: An aggregation theorem for securities markets. J. Financial Econ. 1, 225-244 (1974)

20. Wang, J.: A model of intertemporal asset pricing under asymmetric information. Rev. Financial Stud. 60, 249-282 (1993)

21. Wang, J.: A model of competitive stock trading volume. J. Political Econ. 102, 127-168 (1994)

22. Wang, J.: The term structure of interest rates in a pure exchange economy with heterogeneous investors. J. Financial Econ. 41, 75-110 (1996) 